\title{
Systematic optimization of the storage capacity of slow light photonic crystal waveguides.
}

\author{
Panagiotis Kanakis $^{\mathrm{a}}$, Thomas Kamalakis ${ }^{\mathrm{b}}$, Thomas Sphicopoulos ${ }^{\mathrm{a}}$ \\ ${ }^{a}$ National and Kapodistrian University of Athens, Panepistimioupolis, Athens, Greece GR157 84; \\ ${ }^{\mathrm{b}}$ Department of Informatics and Telematics, Harokopio University, Athens, 89 Harokopou str., \\ Athens, Greece GR176 71.
}

\begin{abstract}
A systematic design process of slow light photonic crystal slab waveguides is presented with the aim of maximizing the storage capacity. Dispersion effects and propagation losses characteristics are included in order to increase the design accuracy. Our procedure allows the optimization of the structure at the same time by varying as many as ten design parameters. We show that storage capacities of almost $32 \mathrm{bits}$ at $40 \mathrm{~Gb} / \mathrm{s}$ and $65 \mathrm{bits}$ at $100 \mathrm{~Gb} / \mathrm{s}$ can be obtained.
\end{abstract}

Keywords: Photonic Crystals, Storage capacity, Optimization.

\section{INTRODUCTION}

Integrated delay lines are considered as one of the cornerstones for the realization of future all optical networks [1]. Slow light nanophotonic structures such as photonic crystals ( $\mathrm{PhCs}$ ) or coupled resonator optical waveguides are attracting increased attention, combining large slowdown factors, sub-wavelength light confinement and enhanced nonlinear effects [2]. There are however, two important factors currently limiting the application of $\mathrm{PhC}$ waveguides (PCWs) as delay lines. Dispersion-induced pulse broadening and propagation losses are both usually enhanced in the slow light regime causing severe signal distortion. Many authors show how to decrease the dispersion and loss characteristics of PCWs by means of design reconfiguration [3]-[5]. By altering a few of the design parameters they are able to come up with low-loss, low-dispersion structures using methods based on exhaustive search. Although using exhaustive search methods, one can obtain a good understanding on how the altered parameters affect slow light propagation, it is difficult, if not impossible, to carry out an exhaustive search if more than two design parameters are involved in the process. Increasing the number of design parameters simultaneously considered in an exhaustive search results a dramatic increase of the computational time. As an alternative, we consider an optimization method that is much less time consuming when more optimization variables are included. In this work, we present a systematic optimization procedure of $\mathrm{PhC}$ waveguides that is significantly faster. In our calculations we realistically include the effects of the dispersion and the loss characteristics of the $\mathrm{PhC}$ waveguides, in an attempt to obtain more meaningful designs from a practical point-of-view. Through the proposed method, the optimum designs for $40 \mathrm{~Gb} / \mathrm{s}$ and $100 \mathrm{~Gb} / \mathrm{s}$ are obtained highlighting the design parameters with the most significant bearing. As we will show further below, an almost threefold increase in storage capacity is obtained for both data rates mentioned above.

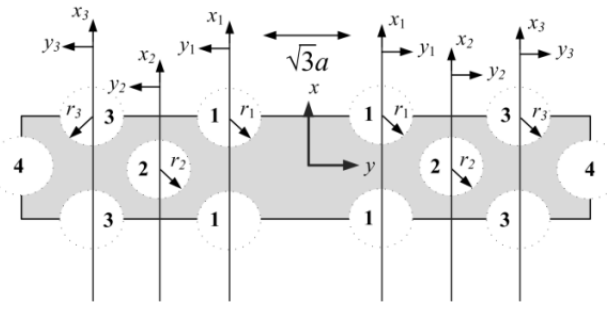

Figure 1. A horizontal cross-section of the PCW illustrating the different design parameters used by the optimization process. The arrows indicate the positive dislocation. The gray filled area is the high index material and the circles are supposed to be filled with air. The holes are classified into groups according to their proximity to the line defect. A number is assigned to each group indicating the proximity level.

Integrated Photonics: Materials, Devices, and Applications II, edited by Jean-Marc Fédéli, Laurent Vivien, Meint K. Smit, Proc. of SPIE Vol. 8767, 876702 · @ 2013 SPIE · CCC code: 0277-786X/13/\$18 · doi: 10.1117/12.2016896 


\section{SYSTEMATIC APPROACH}

The most frequently used figure of merit concerning the buffering capacity of a delay line is the delay bandwidth product (DBP). Although the DBP is a very useful figure of merit, it cannot be directly translated to the storage capacity of a delay line at a given bit rate. Instead in this work we adopt the actual storage capacity of the waveguide $N_{\text {max }}$ estimated by:

$$
N_{\max }=L_{W} R_{b} /\left|v_{g}\right|
$$

where $L_{W}$ is the waveguide length and $v_{g}$ is the group velocity of the defect mode that carries the signal. Here, an aircladding $\mathrm{PhC}$ waveguide formed in a triangular lattice of air holes embedded in a high index material (Si) is considered. The air-holes are classified according to their proximity to the waveguide defect, i.e. class "1" corresponds to the neighbors closest to the waveguide defect, as shown in Figure 1. The figure depicts the PhC waveguide cross section highlighting many of the design parameters considered in this work. We assume that the holes of each class are placed symmetrically with respect to the x-axis. The design parameter $x_{\mathrm{i}}$ allows the dislocation of the holes of the $i$-th class located to the opposite sides of $x$-axis, either in the same [5] or in the opposite direction [4]. The dislocation perpendicular to the waveguide defect is described by the $y_{\mathrm{i}}$ design parameter. The radii of the $i$-th class holes $r_{i}$, as well as the slab height $h$, are also considered in the optimization process. The storage capacity $N_{\max }$, shown in (1) is a function of all these parameters and is formally written as:

$$
N_{\max }=f\left(L_{W}, R_{b}, a, k, r_{\alpha}, \varepsilon_{\alpha}, \varepsilon_{b}, h, x_{1}, y_{1}, r_{1}, \ldots, x_{N}, y_{N}, r_{N}\right)
$$

In (2), $a$ is the lattice constant, $k$ is the wavevector, $h$ and $r_{\alpha}$ are the slab height and radii of the holes not belonging to any class. Finally, $N$ is the number of classes considered in the optimization process, $\varepsilon_{\alpha}$ and $\varepsilon_{b}$ are the relative dielectric constants of the high- and low-index materials. Consequently, the standard W1 waveguide will be obtained by assuming that all of the design parameters are set to zero, except of the design parameter $r_{\mathrm{i}}$ which is equal to $r_{a}$. The function $f$ in (2) is not known in closed form but can be numerically estimated from the dispersion relation, $k=k(\omega)$ and the modal fields of the PhC guided mode. In this work we have implemented a 3D plane wave expansion (PWE) mode solver based on the conjugate gradient of the Rayleigh-Ritz quotient [6], providing us with the dispersion relation and the modal fields. Applying standard optimization methods we are able estimate the arguments of $f$ that provide the maximum $N_{\max }$. In order to locate a maximum value of $N_{\max }$, we have implemented a minimization process based on MATLAB's fmincon function, which is based on an interior-point approach and combines a direct method for solving the constrained minimization problem along with conjugate gradient steps using trust regions [7]. Like any local optimization procedure, this approach is strongly dependent on the initial guess used in the beginning of the optimization. In order to ensure that the maximum value $N_{\max }$ is achieved, we consider restarting the optimization process using different initial points, a technique that is commonly used in such situations. Below we discuss in more detail the estimation of $N_{\max }$, taking into account the performance of state-of-art fabrication techniques [8].

\subsection{Pulse Broadening and Propagation Loss}

As discussed above, the design must respect two fundamental restrictions: the first refers to the maximum tolerable optical power loss level $l_{\max }$ while the second refers to the maximum broadening factor $B_{\max }$. In this work we assume a value of $l_{\max }(\mathrm{dB})=-20 \mathrm{~dB}$, that can easily compensated by semiconductor optical amplifiers [9]. Considering that the loss coefficient $\Gamma$ is known, the maximum length, above witch the propagation losses will be considered prohibitive can be determined by:

$$
L_{\Gamma}=-l_{\max } / \Gamma
$$

In (3), $\Gamma$ is the loss coefficient in $\mathrm{dB} / \mathrm{cm}$. In order to estimate the loss coefficient $\Gamma$ we have implemented the loss model proposed in [3] according to which $\Gamma=c_{1} \gamma n_{g}+c_{2} \rho n_{g}{ }^{2}$ where $\gamma$ and $\rho$ are the out-of-plane and backscattering coefficients, respectively and $n_{g}$ is the group index. The parameters $c_{1}$ and $c_{2}$ are technological parameters that considered independent of the design. The estimation of $\rho$ and $\gamma$ is carried out using the modal fields of the PhC guided mode with the expressions outlined in [3],[10]. For the waveguide designs considered below we have verified that backscattering has dominant optical loss contribution. Under this assumption, the propagation loss coefficient $\Gamma\left(n_{g}\right)$ of any waveguide design can be expressed in terms of the loss coefficient $\Gamma_{\text {ref }}\left(n_{g, \text { ref }}\right)$ of a reference waveguide. It can be easily shown that operating at the same $n_{g}$ and neglecting out-of-plane losses one obtains: 


$$
\Gamma\left(n_{g}\right)=\Gamma_{\text {ref }}\left(n_{g, \text { ref }}\right) \frac{\rho}{\rho_{\text {ref }}}\left[\frac{n_{g}}{n_{g, \text { ref }}}\right]^{2}
$$

In our calculation we have used the loss-engineered waveguide proposed in [3] as our reference waveguide, assuming a loss coefficient of $\Gamma_{\text {ref }}\left(n_{g, \text { ref }}\right)$ of $2 \mathrm{~dB} / \mathrm{cm}$ in the fast light regime $\left(n_{g, \text { ref }}=5\right)$. We have shown in [10] that equation (4) agrees well with experimental results. Although we have excluded the contribution of the out-of-plane losses, we have also verified that the out-of-plane inclusion will only cause a marginal difference to the storage capacity of the waveguide [11]. Alternative loss models however, can be considered as long as they are not too time consuming.

Along with the propagation loss, the pulse broadening in the time domain must also be acknowledged. The dispersion induced broadening is defined as the ratio of the root-mean-square (RMS) width of the pulse at a given position, $\sigma_{L}$ to the incident RMS width, $\sigma_{0}$. The broadening factor is given by [12]:

$$
B F=\frac{\sigma_{L}}{\sigma_{0}}=\left[1+\left(\frac{\beta_{2} L_{W}}{2 \sigma_{0}^{2}}\right)^{2}+\frac{1}{2}\left(\frac{\beta_{3} L_{W}}{4 \sigma_{0}^{3}}\right)^{2}\right]^{1 / 2}
$$

In (5), $\beta_{2}$ and $\beta_{3}$ are the group velocity dispersion (GVD) and the third order dispersion (TOD), respectively. Treating this feature as a length limitation as well, the maximum allowable length due to dispersion will be determined by:

$$
L_{B}=K\left(B_{\max }^{2}-1\right)^{1 / 2}\left(\beta_{2}^{2} R_{b}^{4}+\frac{1}{4} K^{-1} \beta_{3}^{2} R_{b}^{6}\right)^{-1 / 2}
$$

where $K=0.0224$. Here, Gaussian pulses with initial full width at half maximum $T_{F}=1 /\left(4 R_{b}\right)$ and initial RMS width $\sigma_{0} \cong T_{F} / 2.36$, are assumed. The maximum tolerable broadening factor is considered $B_{\max }=1.33$ implying additional $33 \%$ broadening with respect to the incident pulse width [9]. The GVD and TOD can be easily calculated through polynomial fitting of the guided mode dispersion relation. Considering that the waveguide should be realized in integrated form a third restriction arises from the fact that its length cannot exceed a maximum value, $L_{\max }$. In this work we assume that $L_{\max }=1 \mathrm{~cm}$. The final choice of the $L_{\mathrm{W}}$ will be the minimum between those three lengths $\left(L_{\Gamma}, L_{\mathrm{B}}\right.$ and $\left.L_{\max }\right)$. Consequently, selecting the waveguide length in order to satisfy the aforementioned considerations we guarantee that the optimized design will respect all the above limitations.

\section{RESULTS AND DISCUSSION}

We first compare the results obtained by the systematic optimization process starting from designs already proposed in the literature. Figure 2(a) and (b) illustrate the values of $N_{\max }$ with respect to the wavevector for two designs proposed in the literature [3], [4] for a bit rate of $R_{b}=40 \mathrm{~Gb} / \mathrm{s}$. The resulting $N_{\max }$ for the proposed designs at a higher bit rate of $R_{b}=100 \mathrm{~Gb} / \mathrm{s}$ is shown in Figure 2(c) and (d). In the same figures the optimized designs is depicted altering the same
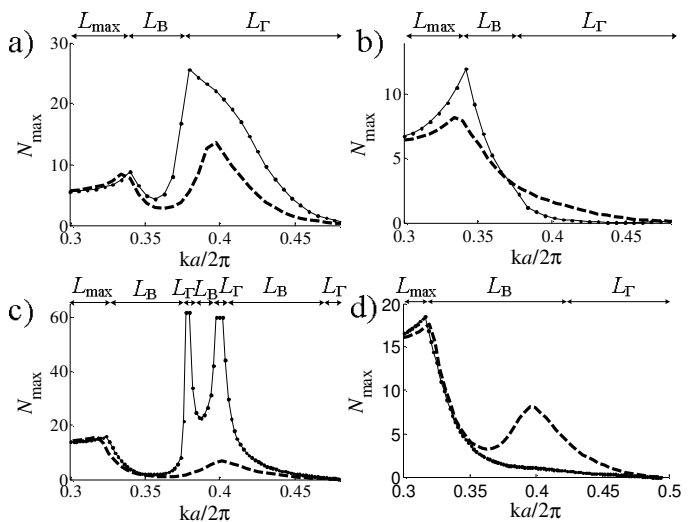

Figure 2. The storage capacity of the original designs (dashed line) proposed by O'Faolain et al. [3] and Liang et al. [4] for $40 \mathrm{~Gb} / \mathrm{s}$ in (a) and (b) and for $100 \mathrm{~Gb} / \mathrm{s}$ in (c) and (d), respectively. In each case the design obtained from the optimization process altering the same design parameters is shown (solid line). 
design parameters as in the proposed waveguides. As depicted in Figure 2(a) and (c), the design proposed by O'Faolain et al. in [3], realized by shifting the first and second class of holes along the $y$-direction by $y_{1} / a=0.1171$ and $y_{2} / a=-0.039$ provides a storage capacity of $N_{\max }=13.4 \mathrm{bits}$ and $N_{\max }=15.8$ bits at $R_{b}=40 \mathrm{~Gb} / \mathrm{s}$ and $R_{b}=100 \mathrm{~Gb} / \mathrm{s}$, respectively. Figure 2 (b) and (d), show the values of $N_{\max }$ with respect to the wavevector for the design proposed by Liang et al. in [4]. In this case the design is realized by altering the position of the first class holes along the $x$-direction by $x_{1} / a=0.105$ and the second class holes along the $y$-direction by $y_{2} / a=-0.084$, obtaining a value of $N_{\max }=8.3$ bits at $R_{b}=40 \mathrm{~Gb} / \mathrm{s}$ and $N_{\max }=17.6 \mathrm{bits}$ at $R_{b}=100 \mathrm{~Gb} / \mathrm{s}$. Considering the same design parameters in each case, the aforementioned optimization procedure almost definitely result a design with greater storage capacity. Table 1 , show the values of $N_{\max }$ for the optimized design in every case, including the design specifications and the obtained group index. As will discuss further below the improvement of the storage capacity strongly depends on the selected design parameters.

Table 1. The optimized designs altering the same design parameters as proposed in [3] and in [4], for the bit rates of $40 \mathrm{~Gb} / \mathrm{s}$ and $100 \mathrm{~Gb} / \mathrm{s}$. The value of Nmax as well as the corresponding group index is also shown.

\begin{tabular}{|c|c|c|c|c|c|c|c|c|}
\hline & \multicolumn{3}{|c|}{ Optimized design based on [3] } & \multicolumn{3}{|c|}{ Optimized design based on [4] } \\
\hline$R_{b}$ & $y_{1} / a$ & $y_{2} / a$ & $N_{\max }(\mathrm{bit})$ & $n_{g}$ & $x_{1} / a$ & $y_{2} / a$ & $N_{\max }(\mathrm{bit})$ & $n_{g}$ \\
\hline $40 \mathrm{~Gb} / \mathrm{s}$ & 0.1354 & 0.0436 & 25.6 & 20 & 0.0302 & -0.0287 & 12 & 9 \\
\hline $100 \mathrm{~Gb} / \mathrm{s}$ & 0.1373 & 0.0561 & 61.7 & 20 & -0.0838 & -0.016 & 18.5 & 5.5 \\
\hline
\end{tabular}

As shown in Figure 2 and in Table 1, considering the $y_{1}$ and $y_{2}$ design parameters the optimization process yield an improvement of the storage capacity by an additional 12.2 bits compared to the proposed design in [3] for $R_{b}=40 \mathrm{~Gb} / \mathrm{s}$ and an additional $45.9 \mathrm{bits}$ for $R_{b}=100 \mathrm{~Gb} / \mathrm{s}$. Considering the $x_{1}$ and $y_{2}$ design parameters, the improvement of the maximum storage capacity is yielding an additional 3.7bits for $R_{b}=40 \mathrm{~Gb} / \mathrm{s}$ and a marginal improvement of additional 0.9 bits for $R_{b}=100 \mathrm{~Gb} / \mathrm{s}$. The wavevector intervals for which the waveguide length of the optimum design is limited either by dispersion effects $\left(L_{\mathrm{W}}=L_{\mathrm{B}}\right)$, by propagation losses $\left(L_{\mathrm{W}}=L_{\Gamma}\right)$ or by the maximum length limitation $\left(L_{\mathrm{W}}=L_{\max }\right)$ is highlighted in Figure 2. The optimum waveguide length is limited by the propagation losses in the cases illustrated in Figures 2(a) and (c) while the dispersion limitation $L_{\mathrm{B}}$ is the contributing factor restraining the waveguide length for the cases shown in Figures 2(b) and (d). We have verified that each optimized design shown in Figure 2, support monomode operation.

Thus far we have applied the optimization process for a few waveguide designs proposed in the literature by considering only the design parameters used in these studies. We will now apply the optimization scheme in order to design a slow light waveguide from scratch considering multiple design parameters. We choose as our starting point a standard W1 waveguide with $r_{\alpha}=0.27 a$ and $h=0.5 a$. We then perform a step-by-step optimization process gradually increasing the number of parameters considered. Figure 3(a) and (b) illustrate the most important optimization steps for $R_{b}=40 \mathrm{~Gb} / \mathrm{s}$ and $R_{b}=100 \mathrm{~Gb} / \mathrm{s}$, respectively. In Figure 3, the capacity of the original W1 waveguide as well as the $k$ interval for which the
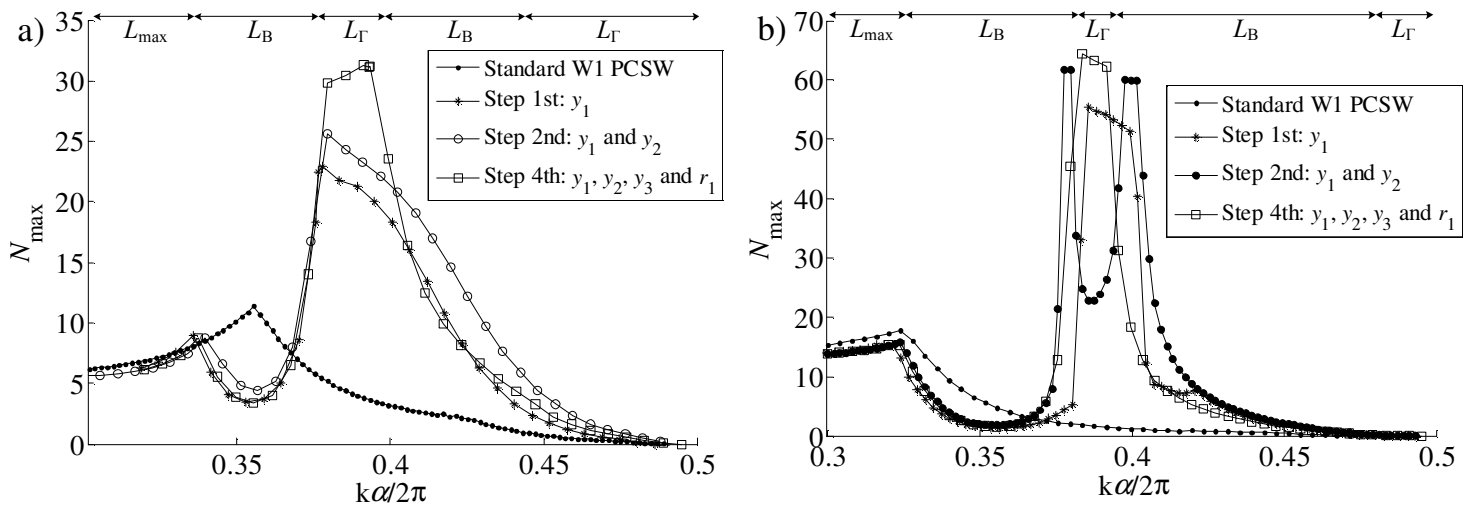

Figure 3 The most important steps of the step-by-step optimization process for the bit rate of (a) 40Gb/s and (b) $100 \mathrm{~Gb} / \mathrm{s}$. The arrows indicate the wavevector intervals in which the waveguide length is limited by either $L_{\mathrm{B}}, L_{\Gamma}$ or $L_{\max }$. 
waveguide length is limited by the dispersion, loss and integration consideration, are also shown. As illustrated in Figure 3(a) and (b), a value of $N_{\max }=11.4$ bits and $N_{\max }=17.8$ bits is obtained by the standard W1 PhC waveguide for $R_{b}=40 \mathrm{~Gb} / \mathrm{s}$ and $R_{b}=100 \mathrm{~Gb} / \mathrm{s}$, respectively. The design specifications of the optimization steps, obtaining the most significant improvement of the storage capacity, are shown in the Table 2 and Table 3 for $R_{b}=40 \mathrm{~Gb} / \mathrm{s}$ and $R_{b}=100 \mathrm{~Gb} / \mathrm{s}$, respectively. The optimization steps not depicted in Figure 3 are yielding a negligible improvement on the storage capacity. The results illustrated in Figure 3(a) and (b) are leading us to the conclusion that the design parameters $y_{1}, y_{2}$ and $r_{1}$ are mostly affecting the storage capacity of the waveguide regardless the applicable bit rate. In Figure 3 , arrows indicate the wavevector intervals in which the waveguide length is limited by either $L_{\mathrm{B}}, L_{\Gamma}$ or $L_{\max }$. It is shown that the waveguide length of the optimum design for either bit rates is loss-limited, when the maximum $N_{\max }$ is obtained. The dispersion relation and the group index of the optimized designs are shown in Figure 4 (a) and (b) for $R_{b}=40 \mathrm{~Gb} / \mathrm{s}$ and $R_{b}=100 \mathrm{~Gb} / \mathrm{s}$, respectively. As depicted in Figures 4(a) and (b), the optimized designs are experiencing regions with almost linear dispersion relation causing practically stable group indices (where the variation is less than $10 \%$ around the mean value) within a range of $\Delta \lambda=11 \mathrm{~nm}$ and $\Delta \lambda=15 \mathrm{~nm}$, respectively. In this optimization process every optimization step is carried out in approximately 10 iterations, each requiring 10-15 function evaluations, yielding a total of 1350 function evaluations for a complete nine-step optimization process. The order of the design parameters used in the optimization process does not play a significant role as long as multiple initial guesses are used in each step. We have verified that each optimized waveguide shown in Figure 3, support monomode operation.

Some interesting conclusions can be deduced regarding the selection of the design parameters in the optimization process. At the $n_{g}$ level experienced in the cases described in Tables 1,2 and 3, the concentration area of the modal field is hardly developed to reach the third class of holes, shown in Figure 1. There by, altering the position of the third or higher class of holes will have a negligible impact on the resulting value of $N_{\max }$. Moreover, design parameters altering the position of the holes along the $\mathrm{x}$-direction, like $x_{\mathrm{i}}$, despite the fact that they have a great contribution on the dispersion effects of the waveguide, they seem to have a minor impact on the reduction of the propagation losses keeping $L_{\Gamma}$ at small values. This fact minimizes the improvement on the storage capacity that can be obtained by altering these design parameters, as shown in Figure 2(b) and (d).

Table 2. The most important steps of the optimization process for $R_{b}=40 \mathrm{~Gb} / \mathrm{s}$.

\begin{tabular}{|c|c|c|c|c|c|c|}
\hline & $y_{1} / a$ & $y_{2} / a$ & $y_{3} / a$ & $r_{1} / a$ & $N_{\max }(\mathrm{bit})$ & $n_{g}$ \\
\hline $\mathrm{W} 1$ & 0 & 0 & 0 & 0.27 & 11.4 & 8.6 \\
\hline Step 1 & 0.1345 & 0 & 0 & 0.27 & 22.9 & 25.1 \\
\hline Step 2 & 0.1354 & 0.0436 & 0 & 0.27 & 25.6 & 19.8 \\
\hline Step 4 & 0.1297 & -0.0248 & 0.399 & 0.25 & 31.2 & 24 \\
\hline
\end{tabular}

Table 3. The most important steps of the optimization process for $R_{b}=100 \mathrm{~Gb} / \mathrm{s}$.

\begin{tabular}{|c|c|c|c|c|c|c|}
\hline & $y_{1} / a$ & $y_{2} / a$ & $y_{3} / a$ & $r_{1} / a$ & $N_{\max }(\mathrm{bit})$ & $n_{g}$ \\
\hline $\mathrm{W} 1$ & 0 & 0 & 0 & 0.27 & 17.8 & 5.3 \\
\hline Step 1 & 0.1373 & 0 & 0 & 0.27 & 55.3 & 25 \\
\hline Step 2 & 0.1373 & 0.0561 & 0 & 0.27 & 61.7 & 18.5 \\
\hline Step 4 & 0.1394 & 0.0246 & 0.0181 & 0.2645 & 65 & 20.4 \\
\hline
\end{tabular}

In conclusion, we have presented an optimization method of the storage capacity of slow light PhC slab waveguides. The dispersion effects and the propagation losses are taken into consideration in order to obtain a more feasible optimized design. We have shown that the optimization process manage to obtain designs with improved storage capacity compared to designs already proposed in the literature. The optimization process was also used in order to design a PhC slab waveguide from scratch starting from the standard W1 waveguide. The results highlight the fact that the design parameters $y_{1}, y_{2}$ and $r_{1}$ seem to have a great impact on the improvement of the storage capacity. In both data rates 

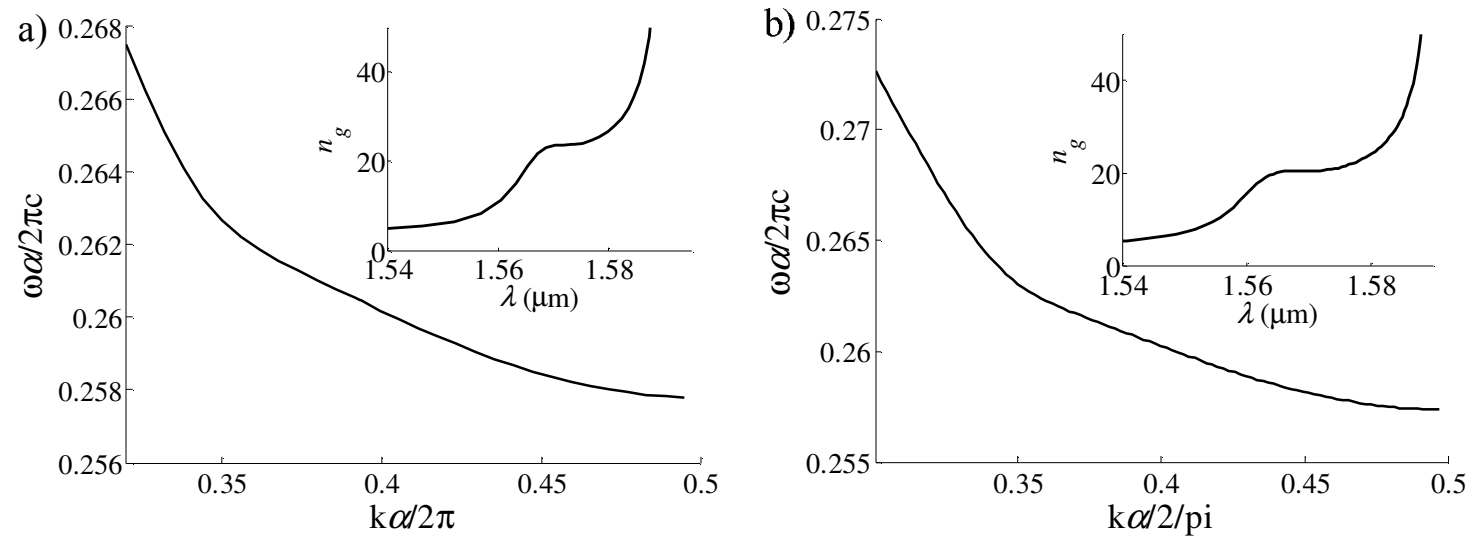

Figure 4. The dispersion relation of the optimum designs shown in Figure 3 for (a) $\mathrm{Rb}=40 \mathrm{~Gb} / \mathrm{s}$ and (b) $\mathrm{Rb}=100 \mathrm{~Gb} / \mathrm{s}$. The group index with respect to the wavelength is also shown in either case.

examined here, the optimization method involving simultaneously ten design parameters, managed to yield a design with a nearly threefold storage capacity compared to the standard $\mathrm{W} 1$ waveguide.

\section{ACKNOWLEDGMENTS}

This research has been co-financed by the European Union (European Social Fund - ESF) and Greek national funds through the Operational Program "Education and Lifelong Learning" of the National Strategic Reference Framework (NSRF) - Research Funding Program: Heracleitus II. Investing in knowledge society through the European Social Fund.

\section{REFERENCES}

[1] Tucker, R. S., Ku, P. C., and Chang-Hasnain, C. J., "Slow-light optical buffers: Capabilities and fundamental limitations," J. Lightw. Technol. 23, pp. 4046-4066 (2005).

[2] Melloni, A., Canciamilla, A., Ferrari, C., Morichetti, F., O’Faolain, L., Krauss, T. F., De La Rue, R., Samarelli, A., and Sorel, M., "Tunable delay lines in silicon photonics: coupled resonators and photonic crystals, a comparison," IEEE Photonics J. 2(2), 181-194 (2010).

[3] O’Faolain, L., Schulz, S. A., Beggs, D. M., White, T. P., Spasenovic, M., Kuipers, L., Morichetti, F., Melloni, A., Mazoyer, S., Hugonin, J. P., Lalanne, P., and Krauss, T. F., "Loss engineered slow light waveguides," Opt. Express 18, 27627-27638 (2010).

[4] Liang, J., Ren, L., Yun, M., Han, X., and Wang, X., "Wideband ultraflat slow light with large group index in a W1 photonic crystal waveguide," J. Appl. Phys. 110, 063103 (2011).

[5] Suzuki, K., and Baba, T., "Nonlinear light propagation in chalcogenide photonic crystal slow light waveguides," Opt. Express 18, pp. 26675-26685 (2010).

[6] Johnson, S. G., and Joannopoulos, J. D., "Block-iterative frequency-domain methods for Maxwell's equations in a planewave basis," Opt. Express 18, pp. 173-190 (2001).

[7] Byrd, R. H., Hribar, M. E., and Nocedal, J., "An interior point algorithm for large-scale nonlinear programming," SIAM J. Optim. 9(4), pp.877-900 (1999).

[8] Baba, T., "Recent progress in on-cip slow light devices," Proc. SPIE 8273, Advances in Slow and Fast Light V, 827319 (Feb. 2012).

[9] Ramaswami, R., and Sivarajan, K. N., [Optical Networks: A Practical Perspective], (Morgan Kaufman, 1998).

[10] Kanakis, P., Kamalakis, T., and Sphicopoulos, T., "Numerical analysis of soliton propagation in photonic crystal slab waveguides for signal processing applications," JOSA B 29, pp. 2787-2796 (2012).

[11] Kanakis, P., Kamalakis, T., and Sphicopoulos, T., "Optimization of the storage capacity of slow light photonic crystal waveguides," Opt. Lett. 37, pp. 4585-4587 (2012).

[12] Agrawal, G. P., [Non-Linear Fiber Optics], 4th edition (Academic 2007). 